\title{
DESIGN, SIMULATION AND FABRICATION OF THERMAL ANGULAR ACCELEROMETERS
}

\author{
Hommood Alrowais $^{l} *$, Oliver Brand ${ }^{l}$, and Pamela T. Bhatti ${ }^{1,2}$
}

${ }^{1}$ School of Electrical and Computer Engineering, Georgia Institute of Technology, Atlanta, GA, USA

${ }^{2}$ School of Medicine, Emory University, Atlanta, GA, USA

\begin{abstract}
This paper introduces a bio-inspired sensor design for detecting angular acceleration in a single plane using thermal convection as a potential sensor platform for a vestibular prosthesis. The working principal of the device is based on probing temperature profile changes along a micro-torus caused by angular acceleration. By properly choosing the locations of the heaters as well as the temperature sensors, the output signal will correlate to in-plane angular acceleration of the microstructure, while canceling out linear acceleration within the plane. Measured devices show a sensitivity of $16.1 \mu \mathrm{V} / \mathrm{rad} / \mathrm{s}^{2}$, and a frequency response of DC to $80 \mathrm{~Hz}$, while consuming a non-optimized 1.1 $\mathrm{mW}$ of power.
\end{abstract}

\section{INTRODUCTION}

In mammals, the vestibular system plays an important role in keeping vision focused and balancing posture. Falls are the leading cause of both fatal and non-fatal injuries for people above the age of 65. Many of these falls are the result of bilateral vestibular loss, which can cause vertigo and imbalance [1]. Although physical rehabilitation is an option for unilateral loss, it is not an option for bilateral vestibular dysfunction. One proposed remedy is a vestibular prosthesis [2,3]. Vestibular prostheses replace balance sensing by electrically stimulating vestibular nerve fibers with electrical currents as resulted by head motion and tilt found in nature. A key element of such vestibular prostheses is the sensing element, which senses angular acceleration. In the present work, we investigate a bio-inspired, thermal angular acceleration sensor for this purpose.

Capacitive and piezoresistive linear accelerometers and gyroscopes are widely used in navigation, consumer electronics, manufacturing and automotive applications [4,5]. The first microfabricated capacitive angular accelerometer was presented by Brosnihan et al [6], with a similar device investigated by O'Brien et al [7]. Both devices operate on the parallel-plate principle using a suspended disk as proof mass. Piezoresistive angular accelerometers were initially developed in [8] using a suspended cantilever to measure angular acceleration. Elkund et al [9] introduced a design that uses the output of four linear accelerometers to discern linear accelerations from rotational ones. Finally, Amarasinghe et al [10] presented a 6-degree of freedom, piezoresistive inertial sensor that detects all three axes of both linear and angular accelerations. By using a proof mass suspended by four tethers, the researchers were able to strategically place piezoresistors to differentiate the motions.

An alternative approach is to use the semi-circular canals (SCCs) of the human vestibular system as inspiration. Arms et al [11] proposed a bio-inspired approach using a pressure sensor to sense the flow in the channel. Ploechinger [12] proposed to use thermal convection to measure the fluid velocity and correlating it to the angular acceleration, but no device was implemented. This method follows principles similar to those explored in thermal linear accelerometers and gyroscopes, first developed in [13].

The device proposed in this work is based on detecting the change in temperature profile along a micro-torus, mimicking a SCC, caused by angular acceleration, similar to [14]. By properly choosing the locations of the heaters as well as the temperature sensors, the output signal will correlate to in-plane angular acceleration of the microstructure. This approach provides a promising sensing platform without any moving parts for medical applications. The basic microstructure follows principles similar to those explored in thermal, linear accelerometers [13], but extends them to angular accelerations.

Consistent with human detection capabilities, the optimal angular accelerometer for a vestibular prosthesis targets a resolution of $1 \mathrm{rad} / \mathrm{s}^{2}$ and a maximum angular acceleration of 175 $\mathrm{rad} / \mathrm{s}^{2}\left(10,000 \mathrm{deg} / \mathrm{s}^{2}\right)$. The device should consume less than 10 $\mathrm{mW}$ and cover a frequency range from $0-20 \mathrm{~Hz}$. The present paper discusses design, fabrication and preliminary characterization of the angular acceleration sensor, and provides design guidelines for optimizing the detection of angular acceleration.

\section{DESIGN AND SIMULATION \\ Design}

The proposed design comprises four or more linear thermal accelerometers that are placed along the circumference of a micromachined torus to detect the tangential acceleration along the torus (Figure 1a). The tangential acceleration relates to the angular acceleration though $\alpha=\mathrm{a} / \mathrm{R}$, where $\alpha$, a, and $\mathrm{R}$ are the angular acceleration, tangential acceleration, and major radius of the torus, respectively. Each of the four linear accelerometers consists of a central heating resistor and two symmetrically arranged resistive temperature probes that monitor the temperature profile generated by the heater.

(a)

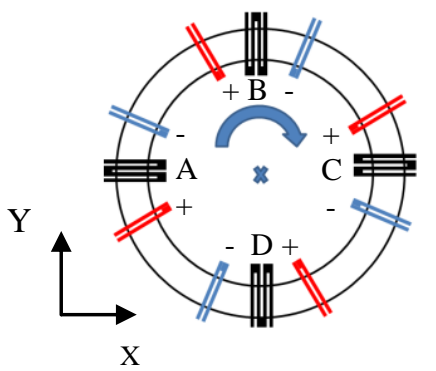

(b)

\begin{tabular}{|c|c|c|c|c|c|c|c|c|}
\hline Acc. & \multicolumn{2}{|c|}{ A } & \multicolumn{2}{|c|}{ B } & \multicolumn{2}{c|}{ C } & \multicolumn{2}{c|}{ D } \\
\hline & + & - & + & - & + & - & + & - \\
\hline$\alpha$ & $\uparrow$ & $\downarrow$ & $\uparrow$ & $\downarrow$ & $\uparrow$ & $\downarrow$ & $\uparrow$ & $\downarrow$ \\
\hline$+\mathrm{X}$ & $\varnothing$ & $\varnothing$ & $\uparrow$ & $\downarrow$ & $\varnothing$ & $\varnothing$ & $\downarrow$ & $\uparrow$ \\
\hline$+\mathrm{Y}$ & $\uparrow$ & $\downarrow$ & $\varnothing$ & $\varnothing$ & $\downarrow$ & $\uparrow$ & $\varnothing$ & $\varnothing$ \\
\hline
\end{tabular}

Figure 1: (a) Basic schematic and operating principle of thermal angular acceleration sensor with four linear thermal accelerometers; (b) Table summarizing the temperature changes of the individual temperature probes as a function of applied acceleration ( $\uparrow:$ temperature increase; $\downarrow$ : temperature decrease, Ø: no temperature change). 
A clockwise angular acceleration applied to the structure increases the temperature of the four red probes, while the four blue probes experience a temperature decrease. Thus, the resulting temperature difference is a measure of the applied (tangential) acceleration. An angular acceleration yields the same output for all four sensors, while linear accelerations can be distinguished by their characteristic response patterns (Figure 1b). In addition, a closed torus structure (similar to the human SCC) is intrinsically insensitive to linear accelerations.

Aluminum is the material chosen for the resistors for its relatively high thermal coefficient of resistance of $3900 \mathrm{ppm} / \mathrm{K}$. The fluid chosen in this work was air at standard conditions. The main property of the fluid that affects the device's performance is the gas diffusivity [15], affecting both the frequency response and the sensitivity of the device. More diffusive fluids such as helium will have a higher bandwidth but a lower sensitivity. Less diffusive fluids such as $\mathrm{CO}_{2}$ or liquids will have higher sensitivity, but a reduced bandwidth.

\section{Finite Element Analysis}

COMSOL $4.3 \mathrm{~b}$ was used to simulate the device using the conjugate heat transfer physics module. Initial device simulations were performed on a 3D linear structure: to this end, the torus was unwrapped and turned into a linear pipe, with periodic boundary conditions for fluid flow and heat transport being applied to the ends of the device, as shown in Figure 2. Subsequently, a comprehensive 3D torus model was simulated.

As an example, Fig. 3 shows the temperature distribution in the center of the pipe around the heating element for the cases of zero applied angular acceleration and $\alpha=1000 \mathrm{deg} / \mathrm{s}^{2}$. From the difference of both temperature profiles, the optimal distance between heater and T-probe can be deduced (Figure 3).
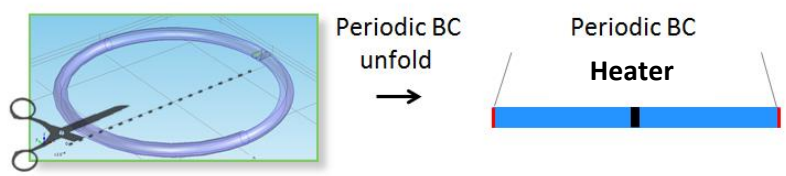

Figure 2: Simplifying the 3D torus structure into a linear pipe with periodic boundary conditions for computational purposes.

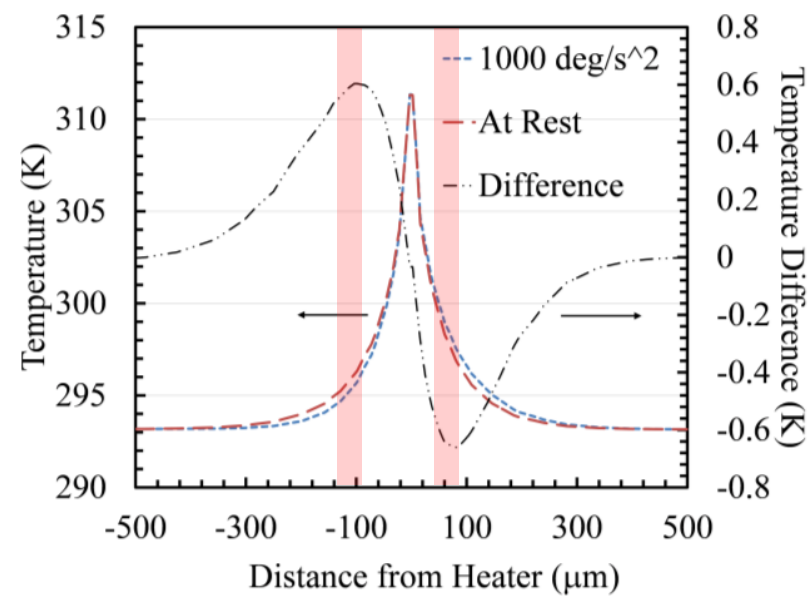

Figure 3: Simulated temperature profile along torus (left and right of a heater) for $\alpha=0$ and $1000 \mathrm{deg} / \mathrm{s}^{2}$ as well as temperature difference between both profiles, showing the optimal location of the temperature sensors highlighted in red.

\section{FABRICATION}

The two-mask fabrication process is schematically highlighted in Figure 4. First, $3400 \AA$ of low-stress PECVD $\mathrm{SiO}_{2}$ is deposited on a silicon wafer (a). Using a lift-off process, a $1200 \AA$ layer of aluminum is sputtered and patterned, forming the resistors and the bonding pads (b). A second mask is used to pattern and etch the oxide film using Deep Reactive Ion Etching (DRIE) (c). Finally, the torus is etched and the $\mathrm{SiO}_{2}$ bridges containing the aluminum resistors are released by isotropic $\mathrm{XeF}_{2}$ etching of silicon $(\mathrm{d})$.

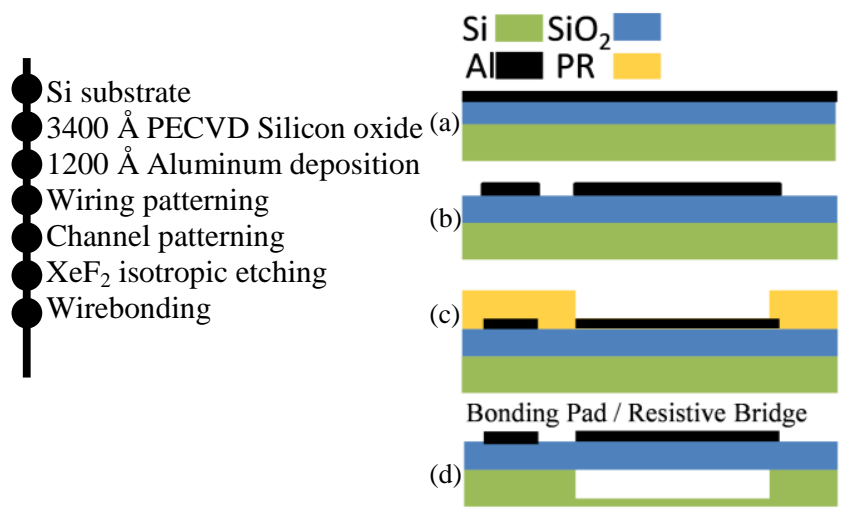

Figure 4: Summary of fabrication process.

Figure 5 shows an optical image of a fabricated angular accelerometer design with $\mathrm{R}=2.85 \mathrm{~mm}$. The inset reveals a different design with $\mathrm{R}=1 \mathrm{~mm}$, having two resistive heaters, and four resistive temperature sensors, each bridging across an etched channel. Different designs were used to study the effect of geometry on sensor performance. Each heater bridge has a length and width of $750 \mu \mathrm{m}$ and $390 \mu \mathrm{m}$, respectively, while the temperatures sensor bridges are $750 \mu \mathrm{m}$ long and $45 \mu \mathrm{m}$ wide. These dimensions were chosen to maximize the temperature elevation in the sensing resistors, thus maximizing device sensitivity.

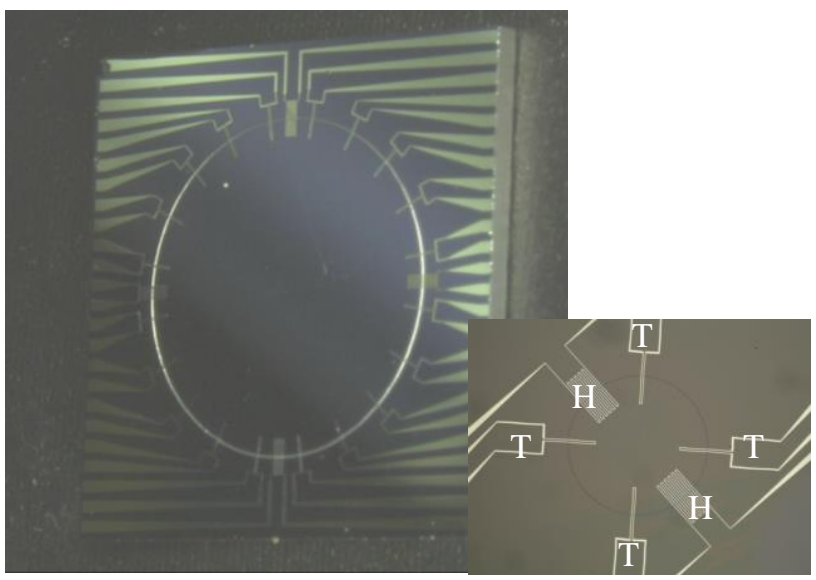

Figure 5: Optical image of fabricated device with $R=2.85 \mathrm{~mm}$, featuring 4 heaters and 20 temperature probes. The inset shows a second design with $R=1 \mathrm{~mm}$, having two heaters $(H)$ and four temperature sensors (T). Each heater bridge has a length and width of $750 \mu \mathrm{m}$ and $390 \mu \mathrm{m}$, respectively, while the temperatures sensor bridges are $750 \mu \mathrm{m}$ long and $45 \mu \mathrm{m}$ wide. 


\section{TESTING}

The angular accelerometers were tested using an Ideal Aerosmith rate table as well as a rotational setup similar to the one described in [14]. The frequency range tested was from DC to $4 \mathrm{~Hz}$, because of setup limitations. A constant $1 \mathrm{~mA}$ current was applied to the heating resistor, while a differential current was applied to the temperature resistors such that a zero differential output voltage across both resistors results at rest. A Stanford Research Systems 830 lock-in amplifier was used to detect the differential output voltage. The time constant was set to $3 \mathrm{~s}$ and the roll-off to $24 \mathrm{~dB}$. The measured voltage from the lock-in amplifier was collected using a Keithley 2636A Sourcemeter. The angular acceleration magnitude was monitored using an Invensense IXZ-500 gyroscope.

\section{RESULTS}

The temperature distribution around the heating resistor was experimentally analyzed using a QFI InfraScope II infrared camera (Figure 6). The base plate with the sample was raised to $60^{\circ} \mathrm{C}$ and used as a reference. A $10 \mathrm{~mA}$ current was supplied to the $550 \Omega$ heating resistor, resulting in a $22.3^{\circ} \mathrm{C}$ temperature elevation in the center of the suspended bridge. The device was at rest during the IR imaging.

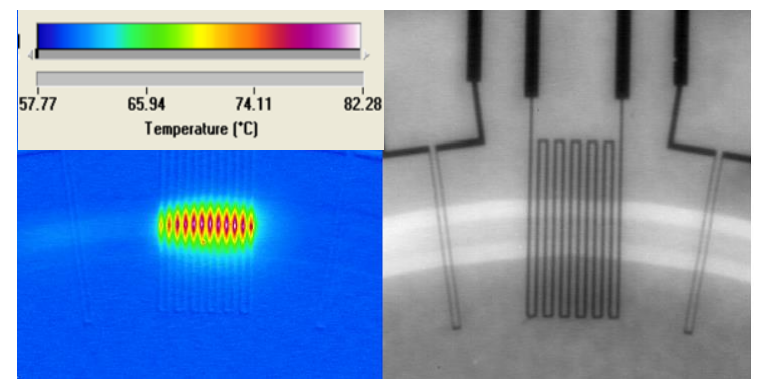

Figure 6: (left) IR image of heater structure with 10mA applied current; (right) Optical image of same heating element with neighboring temperature probes.

Figure 7 shows the response of an example sensor to applied angular acceleration; the particular design tested had an etched channel depth of $350 \mu \mathrm{m}$ and a main radius $\mathrm{R}=2.5 \mathrm{~mm}$. A rate table was used to apply different angular accelerations with a frequency of $2 \mathrm{~Hz}$. The measured device sensitivity was 16.1 $\mu \mathrm{V} / \mathrm{rad} / \mathrm{s}^{2}$. The device consumed $1.1 \mathrm{~mW}$ of power.

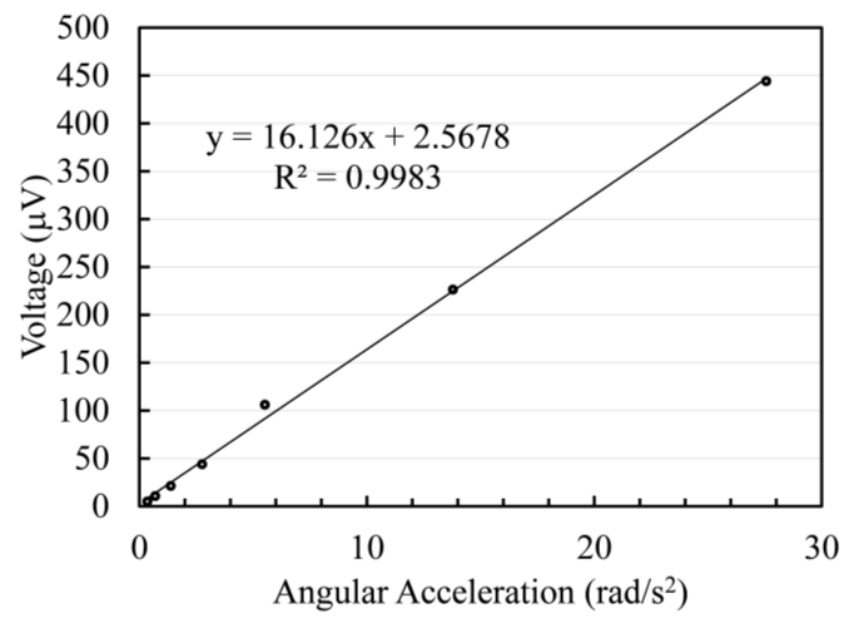

Figure 7: RMS output voltage change across temperature sensor biased with $\operatorname{ImA}$ as a function of the angular acceleration applied $(f=2 \mathrm{~Hz})$. The sensitivity is $16.1 \mu \mathrm{V} / \mathrm{rad} / \mathrm{s}^{2}$.

Due to frequency limitations of the rate table, the frequency response of the device could not be directly measured. Instead of applying a time varying angular acceleration, we applied an ac current directly to the heater and analyzed the frequency dependence of the resulting differential voltage drop across the temperature probes (Figure 8). While this method clearly does not probe the mechanical behavior of the air column inside the torus, but only the frequency dependence of the thermal transport, the resulting frequency characteristic is very similar to FEM results where a tangential acceleration is applied to the gas inside the torus structure. The measured (thermal) cut-off frequency was approx. $80 \mathrm{~Hz}$ for the tested device. The (mechanical) cut-off frequency is proportional to $\sim \chi / \mathrm{r}^{2}$, where $\chi$ is the gas diffusivity, and $\mathrm{r}$ is the minor radius of the channel [15].

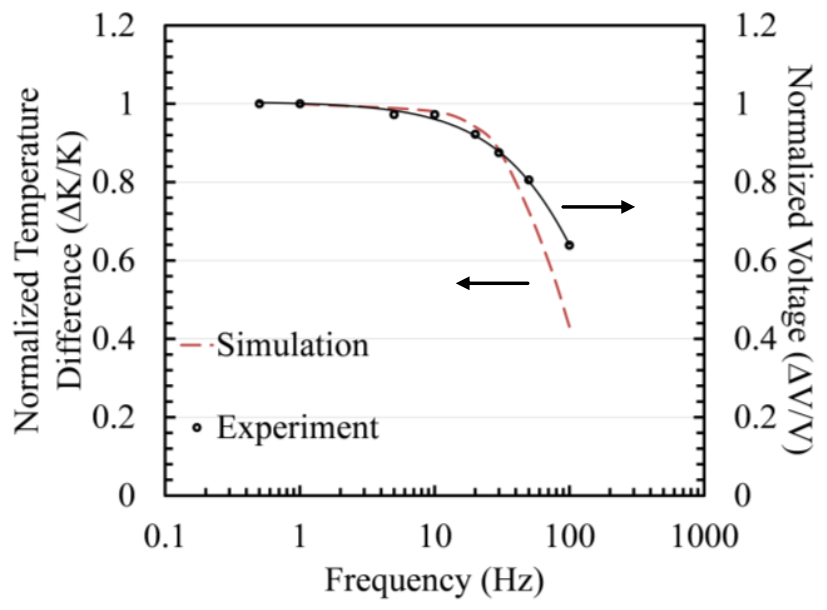

Figure 8: Simulated mechanical and measured thermal frequency transfer characteristic of a thermal angular accelerometer with a major radius of $1.425 \mathrm{~mm}$ and a minor radius of $65 \mu \mathrm{m}$. A peak angular acceleration of $1000 \mathrm{deg} / \mathrm{s}^{2}$ was applied in case of the FEM simulation.

In order to test the ability of the angular acceleration sensors to distinguish between linear and angular accelerations as described in Figure 1b, a sensor with a major radius of $1 \mathrm{~mm}$ and a minor radius of $250 \mu \mathrm{m}$ was tested first in the center of the rate table and then at the edge of the rate table (approx. $20 \mathrm{~cm}$ from the center). While the angular acceleration is the same in both cases, a much larger linear acceleration is superimposed in case of the sensor being mounted off-center (keeping in mind that the rate table undergoes periodic small-angle rotations).

When mounted at the perimeter of the rate table, there is a clear difference between the measured signals of the tangential sensors (1 and 4) as compared to the perpendicular sensors (2 and 3 ), as seen in Figure 9. The difference in sensor output largely stems from the superimposed linear acceleration, which causes a larger signal in the tangentially aligned sensors. If mounted in the center of the rate table, there is a no significant difference between the measured signals of the four sensors, as shown in Figure 10. Thus, it is believed that by proper analysis of the signals coming from the different sensors, linear and angular accelerations can be distinguished. However, the recorded initial measurement data showed unexpected offsets that did not allow for a detailed analysis of the individual signal components. More detailed sensor 
characterization is needed in the future.

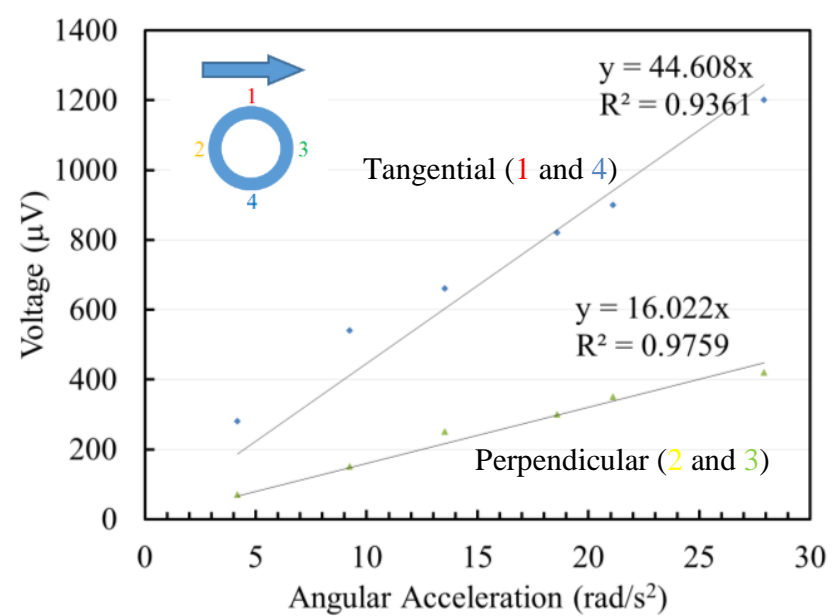

Figure 9: Differential output voltage changes (rms values) across different temperature sensor pairs on the same device as a function of the applied angular acceleration with the sensor being mounted on the edge of the rate table.

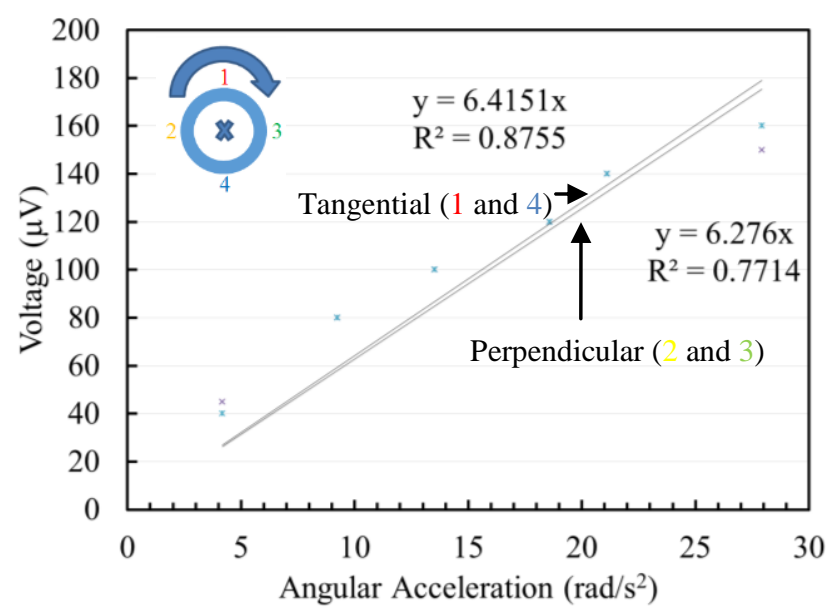

Figure 10: Differential output voltage changes (rms values) across different temperature sensor pairs on the same device as a function of the applied angular acceleration with the sensor being mounted in the center of the rate table.

\section{CONCLUSION}

A thermally sensed, bio-inspired angular accelerometer was designed, simulated, fabricated and tested. Its thermal response was linear to the angular acceleration input within the measured range with a sensitivity of $16.1 \mu \mathrm{V} / \mathrm{rad} / \mathrm{s}^{2}$. The device consumes a non-optimized $1.1 \mathrm{~mW}$ of power.

\section{ACKNOWLEDGMENTS}

This work was supported in part by an NSF CAREER award ECCS-1055801, the National Center for Advancing Translational Sciences of the National Institutes of Health under Award Number UL1TR000454, KL TR000455, and the Saudi Arabian Ministry of Higher Education. Travel support has been generously provided by the Transducers Research Foundation. The authors would like to thank the Georgia Tech IEN cleanroom staff for fabrication assistance, as well as Prof. F. Ayazi, Prof. L. Degertekin, and Prof. I. Papapolymerou for providing access to their lab facilities.

\section{REFERENCES}

[1] J.M. Goldberg, V.J. Wilson, K.E. Cullen, D.E. Angelaki, D. M. Broussard, J. Buttner-Ennever, K. Fukushima, and L.B. Minor, The Vestibular System, A Sixth Sense, Oxford University Press, New York, 2012.

[2] G. Fridman, and C.D. Santina, "Progress Toward Development of a Multichannel Vestibular Prosthesis for Treatment of Bilateral Vestibular Deficiency," The Anatomical Record, vol. 295, (2012) pp. 2010-2029.

[3] D.M. Merfeld, and R.F. Lewis, "Replacing Semicircular Canal Function with a Vestibular Implant", Current Opinion in Otolaryngology \& Head and Neck Surgery, vol. 20, (2012), pp. 386-392.

[4] N. Yazdi, F. Ayazi, and K. Najafi, "Micromachined Inertial Sensors," Proceedings of the IEEE, vol. 86, (1998), pp. 16401659.

[5] B.Chiang, G.Y. Fridman, C.Dai, M.A. Rahman, and C.C. Della Santina, "Design and Performance of a Multichannel Vestibular Prosthesis that Restores Semicircular Canal Sensation in Rhesus Monkey," IEEE Transactions on Neural System Rehabilitation Engingeering, vol. 19, (2011), pp. 588-598.

[6] T.J. Brosnihan, A. Pisano, and R. Howe. "Surface Micromachined Angular Accelerometer with Force Feedback." Digest ASME International Conference (1995), pp. 941-947.

[7] G.J. O'Brien, D.J. Monk, and K. Najafi. "Angular Accelerometer with Dual Anchor Support." TRANSDUCERS, International Conference on Solid-State Sensors, Actuators and Microsystems, (2003), pp. 1371-1374.

[8] Furukawa, Naoyuki, and K. Ohnishi. "A Structure of Angular Acceleration Sensor Using Silicon Cantilevered Beam with Piezoresistors." Industrial Electronics, Control, Instrumentation, and Automation, (1992), pp. 1524-1529.

[9] E.J. Eklund, and A.M. Shkel, "Single-mask Fabrication of High-G Piezoresistive Accelerometers with Extended Temperature Range," Journal of Micromechanics and Microengineering, (2007), pp. 730 .

[10] R. Amarasinghe, D.V. Dao, T. Toriyama, and S. Sugiyama, "Design and Fabrication of Miniaturized Six-Degree of Freedom Piezoresistive Accelerometer," in IEEE International Conference on Micro Electro Mechanical Systems (2005), pp. 351-354.

[11] S.W. Arms, and C.P. Townsend, "MEMS-based Angular Accelerometer," Patent, (2003), US 0047002.

[12] H. Ploechinger, "Sensor for Detecting a Rotational Movement or an Angular Acceleration," Patent, (2004), US 6722199.

[13] A.M. Leung, J. Jones, E. Czyzewska, J. Chen, and B. Woods, "Micromachined Accelerometer based on Convection Heat Transfer," in IEEE International Conference on Micro Electro Mechanical Systems, (1998), pp. 627-630.

[14] J. Groenesteijn, H. Droogendijk, M.J. de Boer, R.G.P. Sanders, R.J. Wiegerink, and G.J.M. Krijnen, "An Angular Acceleration Sensor Inspired by the Vestibular System with a Fully Circular Fluid-channel and Thermal Read-out," in IEEE International Conference on Micro Electro Mechanical Systems, (2014), pp.696-699.

[15] J. Courteaud, N. Crespy, P. Combette, B. Sorli, and A. Giani, "Studies and Optimization of the Frequency Response of a Micromachined Thermal Accelerometer," Sensors and Actuators A: Physical, vol. 147, no. 1 (2008), pp. 75-82.

\section{CONTACT}

*Hommood Alrowais, Georgia Institute of Technology, 777 Atlantic Drive NW, Atlanta, Georgia 30332, USA; Tel: +1-404809-5463; E-mail: hommood.alrowais@ gatech.edu 